\title{
Comportement hydrothermique des amidons de 4 variétés de manioc (Manihot esculenta Crantz) cultivées en Côte d'Ivoire
}

\author{
Sidibe DAOUDA ${ }^{1 *}$, Sako ABOUBAKAR ${ }^{2}$, Pierre EZOUA ${ }^{1}$, Chatigre KOUAME ${ }^{1}$, \\ L. I. DALLY ${ }^{3}$ et Séraphin KATI-COULIBALY ${ }^{4}$
}

${ }^{1}$ Laboratoire de Biochimie et Sciences des Aliments (LaBSA), UFR Biosciences Université de Cocody, Abidjan 22 BP 582 Abidjan 22, Côte d'Ivoire.

${ }^{2}$ Laboratoire de Physique de l'Atmosphère et de Mécanique des Fluides, UFR Sciences de la Structure de la Matière et de Technologie, Université de Cocody, Abidjan, 22 BP 582 Abidjan 22, Côte d'Ivoire.

${ }^{3}$ Laboratoire de Pharmacie Galénique, Biopharmacie et de la Législation Pharmaceutique, UFR des Sciences Pharmaceutiques et Biologiques, Université de Cocody, BPV 34 Abidjan 01, Côte d'Ivoire.

${ }^{4}$ Laboratoire de Nutrition et Pharmacologie, UFR Biosciences, Université de Cocody, Abidjan, 22 BP 582 Abidjan 22, Côte d'Ivoire.

*Auteur correspondant,E-mail : daoudas74@yahoo.fr/daoudas74@hotmail.fr

\begin{abstract}
RESUME
Les études menées dans cet article ont porté sur l'hydrolyse enzymatique et acide ménagée, sur la détermination de la teneur en amylose et en amylopectine, sur le gonflement selon la méthode directe et sur la viscosité des amidons d'Attiéké Mossi 2, d'Agbablé 1, de Kétévie et de TA (8), toutes des variétés de manioc cultivées en Côte d'Ivoire. Ces études ont démontré que l'amidon de Kétévie est le plus enclin aux agressions acide et enzymatique. Et que celui d'Attiéké Mossi 2 est le plus résistant à ces facteurs. Concernant l'amylose, l'amidon d'Agbablé 1 possède la teneur la plus élevée (23,39\%) tandis que celui d'Attiéké Mossi 2 dispose de la plus faible teneur $(18,16 \%)$. La détermination du gonflement selon la méthode directe démontre que l'amidon d'Agbablé 1 présente la plus grande capacité d'absorption d'eau (74,26 g d'eau/g d'amidon) tandis que celui de Kétévie gonfle le moins (56,64 g d'eau/g d'amidon). Au niveau de la viscosité, la plus élevée (225,3 cSt) est observée chez l'amidon d'Attiéké Mossi 2 et la moins élevée (155,5 cSt), chez celui de TA (8). Ces observations font de l'amidon de Kétévie le plus intéressant en termes d'une utilisation en tant qu'excipient dans les comprimés. Aussi, conformément à des études préalables, l'amidon d'Agbablé 1 parait selon la présente étude, le plus prédisposé à une forte libération d'eau (synérèse) lors d'une longue conservation, tandis que celui d'Attiéké Mossi 2 est le moins prédisposé à la synérèse.
\end{abstract}

(c) 2009 International Formulae Group. All rights reserved.

Mots clés: Manihot esculenta Crantz, amidon, manioc, cultivés, Côte d'Ivoire.

\section{INTRODUCTION}

Assurer la sécurité alimentaire est un défit auquel l'Afrique est toujours confrontée. C'est pourquoi, la quasi-totalité des projets de recherche ont été orientés vers les aspects agronomiques des plantes afin d'augmenter leur productivité, négligeant ainsi toutes les investigations apportant une plus value aux productions. C'est ainsi que l'amidon, constituant majeur des tubercules, n'a pas bénéficié d'études pouvant le rendre compétitif sur les marchés internationaux. A 
titre d'exemple, seulement $12 \%$ des publications scientifiques sur l'amidon concernent ceux de l'Afrique (Fao, 1998). Aussi, sur les 400 variétés de manioc dont dispose le Centre National de Recherche Agronomique (CNRA), seulement les amidons d'une vingtaine font actuellement l'objet d'études. Cette absence d'investigations scientifiques induit une méconnaissance des intérêts de l'amidon. Pour preuve, en Côte d'Ivoire, les populations, lors de la fabrication de l'Attiéké (aliment très populaire) rejettent l'amidon. Alors que l'Attiéké coûte au maximum 300 FCFA le kilogramme tandis que l'amidon coûte au moins 700 FCFA le kilogramme.

En plus des implications alimentaires de l'amidon, ses applications industrielles sont toutes aussi nombreuses qu'importantes. Notamment, dans l'industrie papetière et pharmaceutique, dans la construction des infrastructures et dans la chimie des plastiques (Massaux et al., 2006). Dans ce dernier domaine, l'amidon peut se substituer aux dérivés du pétrole avec pour avantage de les rendre biodégradables (Massaux et al., 2006). Mais chacune de ces applications sont tributaires de caractéristiques physicochimiques spécifiques. Ces caractéristiques ne peuvent pourtant être décelées chez les amidons qu'à travers des travaux de recherche portant sur celles-ci.

$\mathrm{Au}$ titre des travaux de recherche, Karim et al. (2007) ont montré que le gonflement et le pouvoir épaississant sont des fonctionnalités recherchées chez les amidons en vu d'une utilisation alimentaire. Selon Davies et al. (2008), la capacité de gonflement d'un amidon dépend de sa capacité à fixer des molécules d'eau à travers des liaisons hydrogènes. Et la teneur en amylose et la proportion de ramification de l'amylopectine jouent un rôle majeur dans cette rétention d'eau conformément aux résultats de Tang et al. (2005). Toujours sur le gonflement, Sidibé et al. (2009) ont montré que la méthode de
Leach (1959) sous-estime la capacité d'absorption des amidons. Et que la méthode directe est plus adéquate.

Aussi, Adrian et al. (1996) ont montré que l'amylose est responsable de la rétrogradation et le rassissement des empois d'amidon. Tandis que d'une part, Schmidt et al. (2006) et d'autre part, Davies et al. (2008) ont montré que la rétrogradation est incommode aux denrées alimentaires destinées à une longue conservation.

En investiguant la viscosité, les travaux de Lagarrigue et al. (2008) ont confirmé le caractère non Newtonien du gel d'amidon. Et selon Thire et al. (2003), Cette viscosité est partiellement due à la libération de l'amylose lors du chauffage.

Seul ce type de recherche pourra rendre les amidons tropicaux compétitifs (Fao, 1998). C'est pour étudier les amidons des variétés de manioc disponibles dans son vivier que le CNRA a initié un vaste programme de caractérisation physicochimique de ceux-ci (Kouakou, 2004). La présente étude rentre dans ce cadre et est la suite de celle entamée en 2005 (Sidibé et al., 2005). Son but est donc d'investiguer les caractéristiques physicochimiques des amidons d'Attiéké Mossi 2, d'Agbablé 1, de Kétévie et de TA (8), afin de prédire leurs voies d'utilisation spécifiques.

\section{MATERIEL ET METHODES Matériel biologique}

Le matériel végétal utilisé dans cette étude est constitué d'amidons extraits par voie humide (Amani, 1993) des 4 cultivars de manioc (Manihot esculenta Crantz): Attiéké Mossi 2, Agbablé 1, Kétévie et TA (8). Tous récoltés dans le vivier du CNRA sise au KM 17 , route de Dabou.

Les enzymes utilisées sont celles contenues dans le suc digestif de l'escargot Achatina achatina. Ce suc est extrait selon la méthode de Séa et al. (2008). 
Cinétique de l'hydrolyse acide ménagée des amidons natifs

L'hydrolyse acide ménagée des amidons a été faite à l'aide de l'acide chlorhydrique 2,2 N (Hoover et Vasanthan, 2004). Cette cinétique est estimée par la détermination de la concentration de sucres totaux (Dubois et al., 1965) contenus dans le surnageant de la solution d'un gramme (g) d'amidon et de $10 \mathrm{ml}$ d'acide chlorhydrique placée dans des conditions de température de $35{ }^{\circ} \mathrm{C}$ pendant 30 jours. L'expérience a été répétée 2 fois.

\section{Digestibilité enzymatique des amidons natifs}

La digestion in vitro des amidons natifs a été faite à l'aide du suc digestif de l'escargot Achatina achatina (Sea et al.., 2008). Cette action est appréciée en estimant la quantité de sucres totaux présents dans le milieu digestif. En effet, à 1 g d'amidon natif mis dans un tube, sont ajoutés $100 \mathrm{ml}$ de solution tampon acétate à pH 6 auquel est additionné $0,5 \mathrm{ml}$ de suc digestif de concentration protéique 0,58 $\mathrm{mg} / \mathrm{ml}$. Ensuite, le mélange est maintenu à 37 ${ }^{\circ} \mathrm{C}$ pendant 5 heures. Après centrifugation à 3000 tours/min pendant 5 minutes, le surnageant obtenu a fait l'objet de dosage de sucres totaux. L'expérience a été répétée 2 fois.

Détermination des teneurs en amylose et en amylopectine des amidons étudiés

La détermination des teneurs en amylose et amylopectine est faite selon la méthode de Chratil (1987) modifiée par Freitas et al. (2004). Cette méthode nécessite l'élaboration d'une courbe d'étalonnage.

La courbe d'étalonnage d'amylose et d'amylopectine purs

La gamme de concentration ayant servi à la construction de cette courbe est constituée de la façon suivante: $0,5 \mathrm{~g}$ d'un mélange d'amylose et d'amylopectine purs (produit Sigma), à des proportions d'amylose de 100 ,
$80,70,50,40,20$ et $0 \%$ sont introduits dans des béchers de $100 \mathrm{ml}$ contenant $20 \mathrm{ml}$ d'eau distillée. Après chauffage de ces suspensions pendant 15 minutes à $75^{\circ} \mathrm{C}$, les volumes sont complétés à $100 \mathrm{ml}$ avec de l'eau distillée. A partir de chacune de ces solutions mères, il est effectué un prélèvement de $1 \mathrm{ml}$ qui est introduit dans des tubes à essais. Le contenu de ces tubes est complété à $5 \mathrm{ml}$. Après homogénéisation, $0,1 \mathrm{ml}$ de lugol $\left(\mathrm{I}_{2} / \mathrm{KI}\right)$ est ajouté au contenu de chaque tube à essais. Ensuite, les tubes sont laissés au repos pendant 10 minutes avant la lecture de l'absorbance ou la densité optique (DO) à 595 $\mathrm{nm}$ (Tetchi et al., 2007). Le graphique f $([$ amylose $])=$ DO (amylose) représente la courbe étalon.

Préparation des échantillons d'amidon pour analyse

$10 \mathrm{~g}$ de chaque amidon sont délipidés à l'hexane selon la méthode de Soxhlet (AOAC, 1975) pendant 6 heures. Ces fractions délipidées sont ensuite séchées à l'étuve à 80 ${ }^{\circ} \mathrm{C}$ pendant 3 heures. De chaque fraction délipidée et séchée, il a été prélevé $0,5 \mathrm{~g}$ sur lesquels il est appliqué le même traitement que celui appliqué sur la gamme d'étalonnage. Les DO obtenues sont reportées sur la courbe d'étalonnage d'amylose pour mesurer la teneur en amylose des amidons étudiés. Ensuite, la teneur en amylopectine est déterminée par simple déduction selon la formule suivante :

$\%$ d'amylopectine $=100 \%-\%$ d'amylose

\section{Etude du gonflement des amidons étudiés}

Le gonflement a été étudié à partir de la taille des grains qui sont de forme quasisphérique, selon la méthode directe (Sidibé et al., 2009). Cette méthode considère uniquement l'eau contenue dans les grains et non celle agglomérée à la surface du gel comme dans la méthode de Leach (1959). Elle passe nécessairement par le suivi de l'évolution granulométrique des amidons lors 
du chauffage, afin de déterminer les tailles moyennes maximum acquises par les grains.

Evolution granulométrique des amidons lors du chauffage

La gamme de températures de chauffage va de 60 à $90 \quad{ }^{\circ} \mathrm{C}$ avec un espacement régulier de $5{ }^{\circ} \mathrm{C}$. A chacune de ces températures, la suspension d'amidon (4\% $\mathrm{P} / \mathrm{V})$ préalablement préparée dans l'eau distillée et mise dans un bécher de $150 \mathrm{ml}$ et chauffée pendant 30 minutes dans un bain marie viscosimétrique thermostaté MD-18V à affichage digital (Instrument JULABO). Pour chaque température, une nouvelle suspension est constituée et l'opération est répétée 2 fois. Des prélèvements en vue d'observations microscopiques sont faits pour chaque température, toutes les 5 minutes. Pour chaque période de prélèvement, au moins 100 photos sont capturées à l'aide d'un microscope de marque CETI équipé d'une caméra d'enregistrement TK-C721EG (Instrument JVC). Cet ensemble est piloté par un ordinateur via le logiciel KAPPA. Et sur ces photos, les tailles de 1000 grains sont mesurées grâce au même logiciel KAPPA. Le comportement de l'ensemble des grains présents à cet instant dans le milieu de chauffage est apprécié par les déterminations des tailles moyennes des grains d'amidon.

Détermination du volume moyen des grains natifs (Vmg)

Seul le diamètre moyen des grains natifs est considéré. Ce volume est exprimé conformément à celui d'une sphère par la formule suivante :

$\mathrm{Vmg}=\frac{4}{3} \pi\left(\frac{d}{2}\right)^{3}$

où $d$ est le diamètre moyen de l'amidon natif.

Détermination du nombre de grain par gramme d'amidon natif $\left(\mathrm{N}_{\mathrm{gr} / \mathrm{g}}\right)$

Le gonflement étant exprimé en gramme d'eau/gramme d'amidon, il est nécessaire de déterminer le nombre de grains d'amidon contenu dans un gramme d'amidon.
Ce nombre est déterminé selon la formule suivante :

$\left(\mathrm{N}_{\mathrm{gr} / \mathrm{g}}\right)=\frac{1}{\rho v_{\mathrm{m} g}}(2)$

(1) et $(2) \Rightarrow \mathrm{N}_{\mathrm{gr} / \mathrm{g}}=\frac{6}{\rho \pi}\left(\frac{1}{d}\right)^{3}$

où $\rho$ est la masse volumique de l'amidon

\section{Gonflement (Q) (gramme d'eau/gramme d'amidon)}

Le gonflement des grains d'amidon est donné par la relation de Sidibé et al. (2009):

$\mathrm{Q}=\mathrm{N}_{\mathrm{gr} / \mathrm{g}} \times \mathrm{V}_{\mathrm{Mg}}$ (4), avec

$\mathrm{V}_{\mathrm{Mg}}=\frac{4}{3} \pi\left(\frac{D-d}{2}\right)^{3}$

où $D$ est le diamètre moyen optimum acquis par les grains lors du chauffage.

Finalement,

(3), (4) et $(5) \Rightarrow \quad \mathrm{Q}=\frac{1}{\rho}\left(\frac{D-d}{d}\right)^{3}$

où $\mathrm{Q}$ est exprimé en gramme d'eau/gramme d'amidon si $\rho$ est en $\mathrm{g} / \mathrm{cm}^{3}$ ou $\mathrm{ml}$.

L'application de cette formule nécessite la détermination de la masse volumique de l'amidon. D'où l'étape suivante.

Détermination de la masse volumique des amidons étudiés

La masse volumique $\rho$ de chaque amidon a été déterminée à l'aide d'un pycnomètre à hélium Micromeritics AccuPyc 1330. Une masse M d'amidon est introduite dans le tube du pycnomètre de volume connu V1. Une fois introduit dans le pycnomètre, un volume V2 d'hélium est envoyé dans le tube du pycnomètre jusqu'à son emplissage complet. Ainsi, la masse volumique de l'amidon est donnée par la relation:

$\rho=\mathrm{M} /(\mathrm{V} 1-\mathrm{V} 2)$.

Cet appareil a l'avantage d'éviter le gonflement de la matière à analyser qui survenait avec les pycnomètres à eau. 
Etude de la viscosité des amidons étudiés

La viscosité des amidons a été étudiée à l'aide d'un viscosimètre Visco Elite $\mathrm{R}$ (instrument FUNGILAB). $500 \mathrm{ml}$ d'une solution à $4 \%(\mathrm{p} / \mathrm{v})$ préalablement préparée dans un bécher de $600 \mathrm{ml}$ sont posés dans un bain marie qui lui-même est posé sur un agitateur magnétique pour éviter la sédimentation de l'amidon. La viscosité est étudiée dans une gamme de températures comprise entre 60 à $90^{\circ} \mathrm{C}$ avec un espacement régulier de $5{ }^{\circ} \mathrm{C}$. Le viscosimètre est piloté par un ordinateur via le logiciel Fungilab. Les résultats sont enregistrés grâce au Logiciel Dataloger. Chaque expérience est réalisée 3 fois.

\section{Analyse statistique des résultats obtenus}

La comparaison des valeurs moyennes obtenues a été réalisée en effectuant des analyses de variance à un critère (ANOVA 1). Les tests ont été réalisés à l'aide du logiciel STATISTICA version 6.0. La statistique du test $(\mathrm{F})$ a été comparée à la valeur théorique en utilisant le seuil de signification $\alpha=0,05$.

\section{RESULTATS}

Hydrolyse acide ménagée des amidons étudiés

L'hydrolyse acide ménagée des amidons étudiés croît de façon considérable jusqu'au $25^{\mathrm{e}}$ jour (Figure 1), date à partir de laquelle on observe un ralentissement de celle-ci marqué par une phase stationnaire.

L'étude montre que l'amidon le plus enclin à l'effet de l'acide est celui du cultivar Kétévie, suivi de celui de TA (8), d'Agbablé1 et d'Attiéké Mossi 2; ce dernier est donc le plus résistant à ce facteur. Il n'y a pas de différence significative entre les amidons au seuil de $5 \%$.

L'observation microscopique des grains après le $25^{\mathrm{e}}$ jour présente des grignotements plus ou moins avancés sur les surfaces des grains. Ces grignotements induisent des pores qui sont à l'origine de canaux de corrosion (Gallant et al., 1982).

\section{Digestibilité enzymatique de l'amidon natif}

Les résultats générés par la digestion in vitro des amidons natifs sont inhérents à leur utilisation en tant qu'excipient dans les comprimés. Plus l'amidon natif s'hydrolyse aisément, cela indique une bonne digestibilité, et plus son utilisation dans la fabrication de comprimé est intéressante. Car une fois avalé, l'amidon enrobant les molécules est soumis aux enzymes du tube digestif (Dreher et al., 1984).

L'allure des graphes de la digestibilité (Figure 2) est similaire à celles des courbes de l'hydrolyse acide ménagée. En effet, ces graphes présentent une phase d'ascension rapide suivie d'une phase de ralentissement. Comme l'a montré l'hydrolyse acide étudiée plus haut, l'amidon le plus enclin à l'attaque des amylases contenues dans le suc gastrique est celui de Kétévie tandis que celui d'Attiéké Mossi 2 est le plus résistant à la digestion. L'implication de ces cultivars dans la fabrication de comprimé en termes d'intérêt suit le même ordre que la sensibilité à l'hydrolyse enzymatique. Cette observation n'est pas conforme aux affirmations de Massaux et al. (2006) qui stipulent que plus l'amidon est constitué de petits grains, moins il est enclin aux agressions extérieures. Car ici, l'amidon d'Agbablé 1 qui dispose de la plus grande proportion de petits grains n'est pas le plus résistant. Il n'y a pas de différence significative entre les amidons étudiés au seuil de $5 \%$.

\section{Teneur en amylose et en amylopectine des amidons étudiés}

Les teneurs en amylose des amidons étudiés ont été obtenues à partir d'une courbe étalon dont le coefficient de corrélation est 0,981 .

Les teneurs en amylose des amidons étudiés varient de $18,16 \% \quad( \pm 1,42)$ pour 


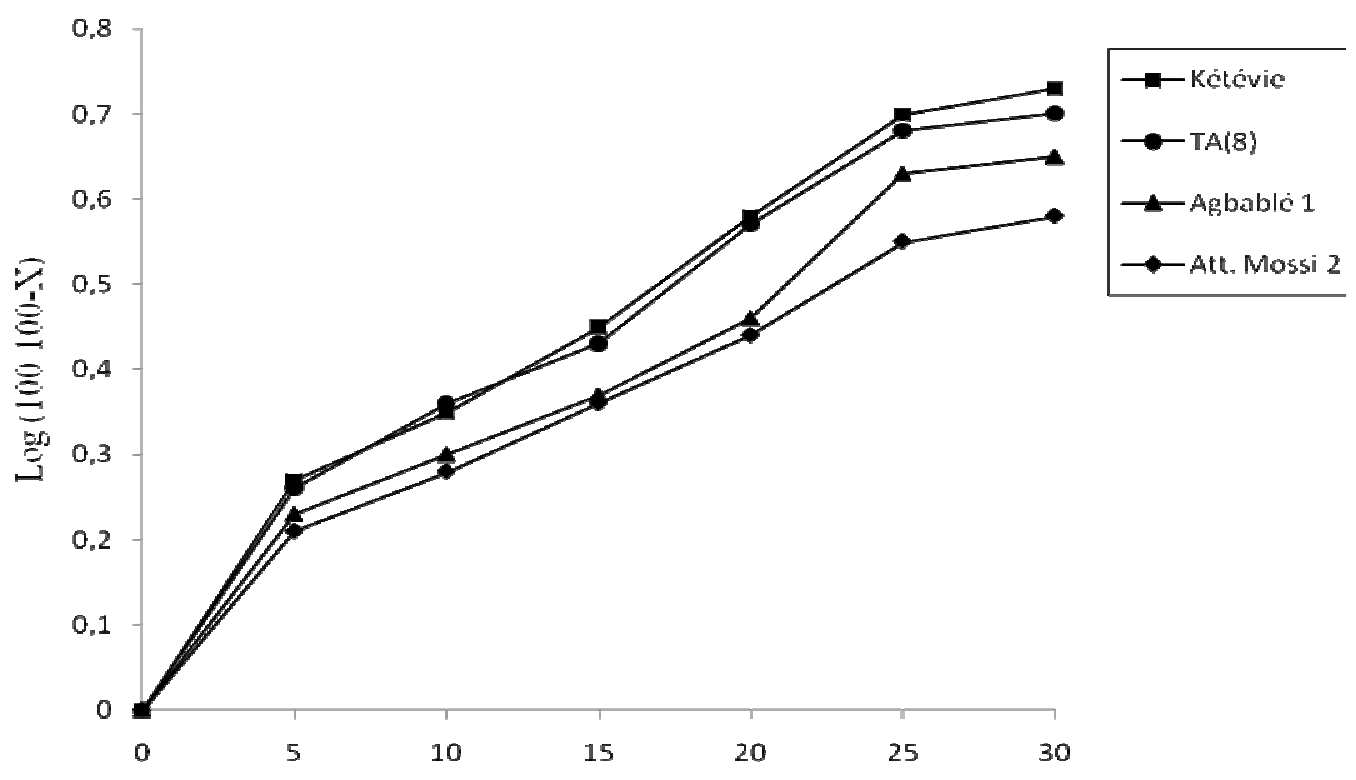

Temps (Jour)

Figure 1: cinétique d'hydrolyse acide ménagée des amidons natifs étudiés pendant 30 jours (X= quantité de sucre hydrolysée ; Att : Attiéké). Les coefficients de variation n'excèdent pas 20\%.

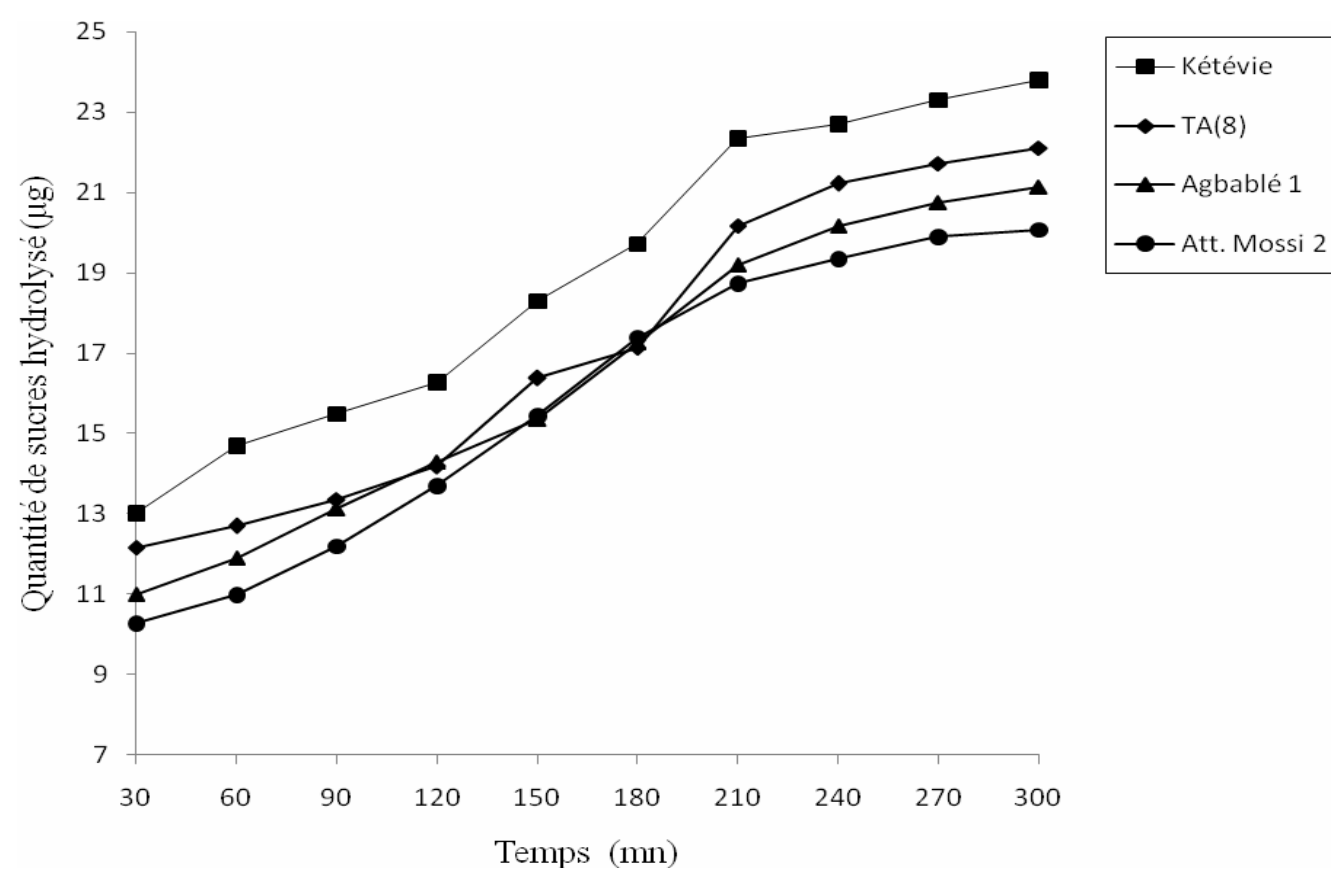

Figure 2: Cinétique d'hydrolyse enzymatique in vitro des amidons natifs étudiés pendant 5 heures (Att : Attiéké). Les coefficients de variation n'excèdent pas $20 \%$. 
l'Attiéké Mossi 2 à 23,39\% ( $\pm 1,19)$ pour Agbablé 1 en passant par 19,33\% ( $\pm 1,26)$ pour TA (8) et 20,03\% ( $\pm 0,85)$. Ce qui signifie que l'amidon d'Attiéké Mossi 2 aura une utilisation plus judicieuse que ceux des autres cultivars étudiés, dans les gelées. Il n’y a pas de différence significative entre les amidons étudiés au seuil de 5\%.

\section{Gonflement des amidons étudiés}

Les gonflements exprimés à travers la figure 3 sont obtenus en utilisant les tailles moyennes maximales des grains d'amidon et les masses volumiques (Tableau 1) des amidons. Les évolutions des gonflements comportent globalement 3 phases.

La première phase est comprise entre $60{ }^{\circ} \mathrm{C}$ et $65^{\circ} \mathrm{C}$. Elle est caractérisée par l'absence de gonflement chez tous les amidons étudiés à l'exception de celui d'Agbablé 1. Cette situation est due à la nonabsorption d'eau par les grains, liée à la résistance opposée par ceux-ci à ces températures. Cependant, la présence de gonflement chez Agbablé 1 est la conséquence de sa sensibilité précoce au choc thermique.

La deuxième phase est comprise entre $65{ }^{\circ} \mathrm{C}$ et $85^{\circ} \mathrm{C}$ pour tous les amidons étudiés à l'exception de celui de TA (8), pour qui, elle se termine à $80 \quad{ }^{\circ} \mathrm{C}$. Cette phase est caractérisée par une forte augmentation du gonflement. Elle correspond aux périodes d'absorptions massives d'eau des grains. Les grains croissent et atteignent 101,88 g d'eau/g d'amidon pour Attiéké Mossi 2; 154,36 g d'eau/g d'amidon pour Agbablé 1 et $56,64 \mathrm{~g}$ d'eau/g d'amidon pour kétévie à $80{ }^{\circ} \mathrm{C}$. Il atteint $74,26 \mathrm{~g}$ d'eau/g d'amidon pour TA (8) à $80^{\circ} \mathrm{C}$.

La troisième phase qui part de la fin de la deuxième à $90{ }^{\circ} \mathrm{C}$ se caractérise par un ralentissement des gonflements dans les milieux de chauffage. Elle correspond à la période où les gros grains gonflés pendant les périodes d'absorption massive ont éclaté. Il reste alors dans les milieux de chauffage, les petits grains qui résistent au choc thermique plus que les gros grains (Massaux et al., 2006). Ainsi, le gonflement apprécié à travers l'eau contenue dans les grains, ralenti et décrô̂t jusqu'à la fin du chauffage.

L'amidon présentant le gonflement le plus élevé est celui d'Agbablé 1 qui est largement au-dessus des autres. Et l'amidon présentant le gonflement le moins élevé est celui de Kétévie. Considérant les teneurs en amylose et conformément aux études antérieures (Tang et al., 2005), il est normal que l'amidon d'Agbablé 1 gonfle le plus. Il y a une différence significative entre l'amidon d'Agbablé 1 et les autres amidons étudiés au seuil de $5 \%$.

\section{Viscosité des amidons étudiés}

L'observation de l'évolution des viscosités nous permet de subdiviser les amidons étudiés en trois groupes (Figure 4).

Le premier groupe, composé de l'amidon d'Attiéké Mossi 2, présente des évolutions en trois phases. La première phase, caractérisée par une croissance lente allant de $25,1 \mathrm{cSt}\left(60{ }^{\circ} \mathrm{C}\right)$ à $28,67 \mathrm{cSt}\left(70{ }^{\circ} \mathrm{C}\right)$. La deuxième phase représente une croissance rapide des viscosités exprimées par des pentes abruptes allant de $28,67 \mathrm{cSt}\left(70^{\circ} \mathrm{C}\right)$ à $206 \mathrm{cSt}$ $\left(75^{\circ} \mathrm{C}\right)$. Enfin, la troisième phase est lente et va de $75^{\circ} \mathrm{C}$ jusqu'à la fin du chauffage où la viscosité atteint 225,3 cSt; $\quad(\mathrm{cSt}=$ centiStockes).

Le deuxième groupe est composé uniquement de l'amidon de TA (8). Sa viscosité croît régulièrement lors du chauffage pour atteindre $155,5 \mathrm{cSt}$ à $90^{\circ} \mathrm{C}$.

Le dernier groupe est composé des deux autres amidons. Leurs viscosités évoluent en deux phases. La première phase est une croissance rapide et caractérisée par une pente abrupte allant de $53,1 \mathrm{cSt}\left(60{ }^{\circ} \mathrm{C}\right)$ pour Agbablé 1 et de $44,9 \mathrm{cSt}\left(60^{\circ} \mathrm{C}\right)$ pour Kétévie à $221,93 \mathrm{cSt}\left(70^{\circ} \mathrm{C}\right)$ pour Agbablé 1 et à $89,1 \mathrm{cSt}\left(70^{\circ} \mathrm{C}\right)$ pour Kétévie. La dernière 
phase représente des viscosités à croissance lente jusqu'à la fin du chauffage où elles atteignent respectivement 221,33 cSt et 221,3 cSt. Ces mêmes comportements sont observés sur la bouillie des nourrissons (Dillon, 1989).
Les résultats obtenus montrent que la viscosité la plus élevée est observée chez l'amidon d'Attiéké Mossi 1 (227,07 cSt) et la moins élevée, chez celui de TA (8) (155,5 cSt). Il y a une différence significative entre TA (8)

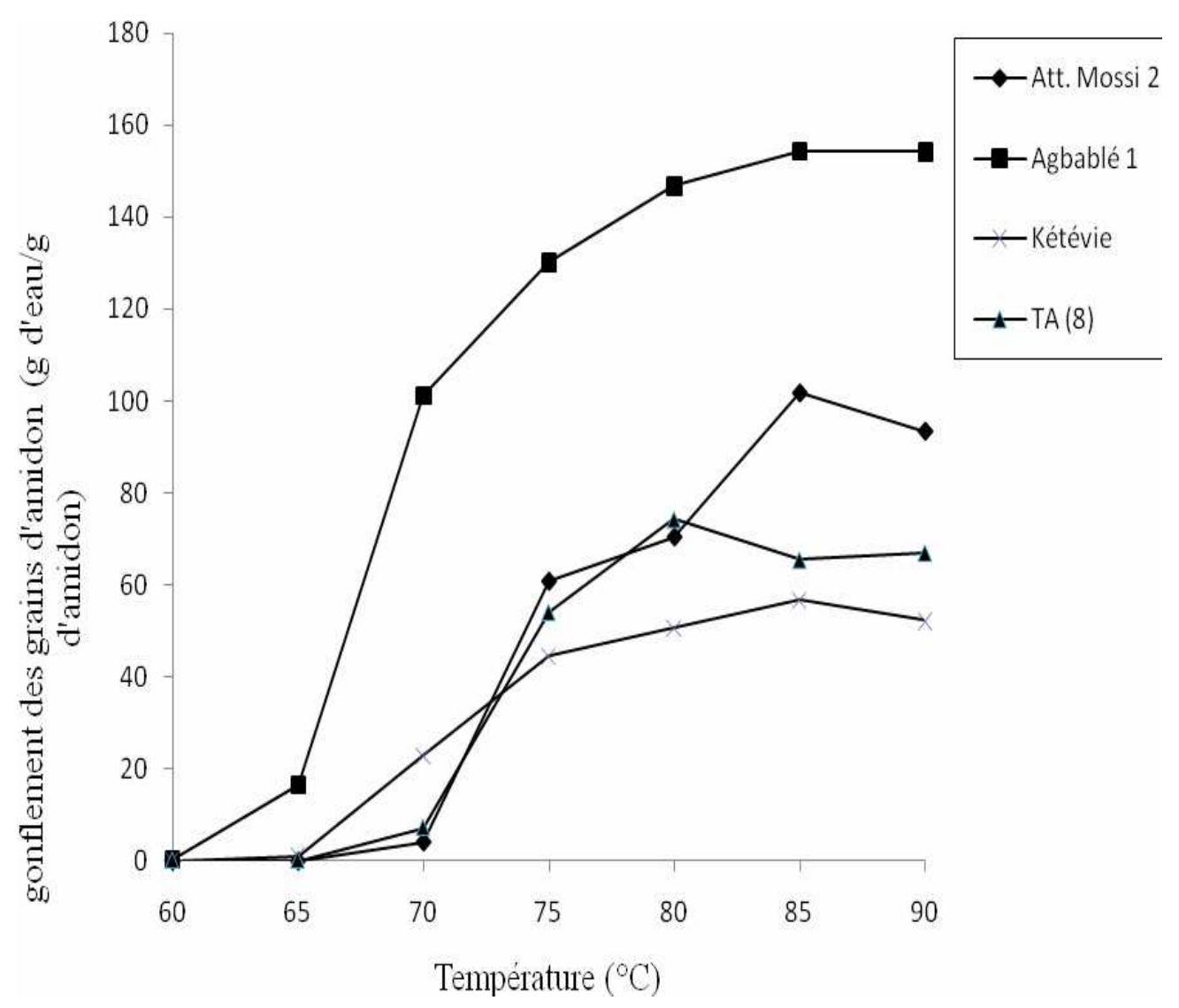

Figure 3: évolution du gonflement des amidons étudiés en fonction de la température et selon la méthode directe (Att : Attiéké). Les coefficients de variation n'excèdent pas 20\%.

Tableau 1 : Masses volumiques des amidons étudiés.

\begin{tabular}{|c|c|c|c|c|}
\hline & Attiéké Mossi 2 & Agbablé 1 & Kétévie & TA (8) \\
\hline $\begin{array}{l}\text { Masses } \\
\text { volumiques } \\
\left(\mathrm{g} / \mathrm{cm}^{3}\right)\end{array}$ & $1,5149 \pm 0,0013^{\mathrm{a}}$ & $1,5040 \pm 0,0025^{\mathrm{a}}$ & $1,5109 \pm 0,0033^{\mathrm{a}}$ & $1,5127 \pm 0,0018^{a}$ \\
\hline
\end{tabular}




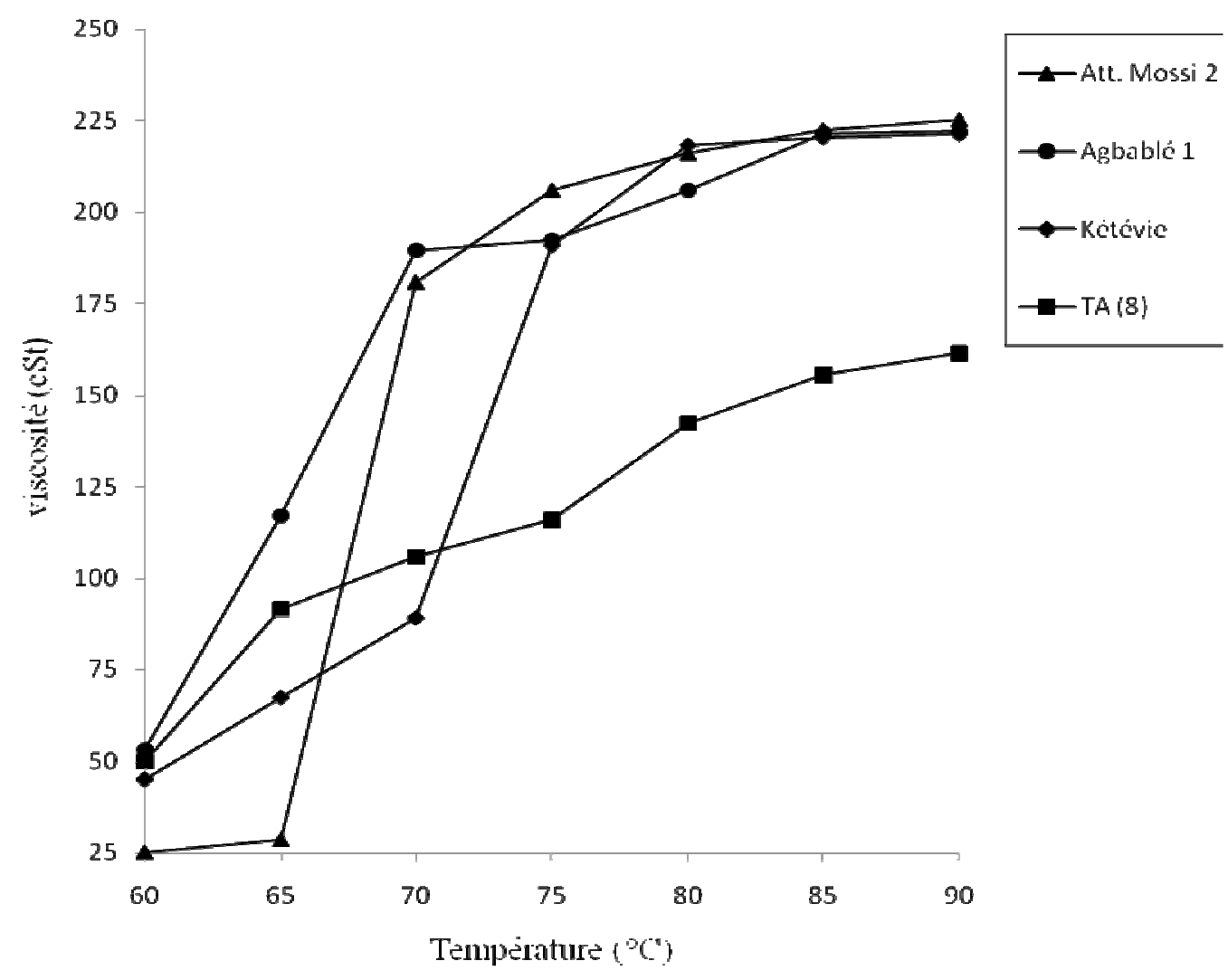

Figure 4: Evolution de la viscosité des amidons étudiés (Att : Attiéké). Les coefficients de variation n'excèdent pas $20 \%$.

et les autres, pendant qu'il n'y a pas de différence significative entre les 3 autres amidons au seuil de $5 \%$.

\section{DISCUSSION}

Hydrolyse acide ménagée des amidons étudiés

L'ordre de sensibilité observé dans cette étude n'est pas conforme aux affirmations de Massaux et al. (2006) qui stipulent que plus l'amidon est constitué de petits grains, moins il est enclin aux agressions extérieures. Car ici, l'amidon d'Agbablé 1 qui dispose de la plus grande proportion de petits grains n'est pas le plus résistant.
L'allure de l'évolution observée sur les amidons natifs de cette étude est la même que celle observée chez celui du gingembre (Amani et al., 2004). Mais dans le cas du gingembre, la phase stationnaire débute à partir du $15^{\mathrm{e}}$ jour, après avoir atteint un niveau d'hydrolyse plus élevé comparativement aux nôtres. Ce qui signifie que l'amidon du gingembre s'hydrolyse plus vite que ceux étudiés dans ce travail. Les mêmes observations sont faites également sur les amidons du blé, de la patate et de l'igname (N'guessan, 2007).

Conformément à l'explication d'Amani et al. (2004), les phases ascendantes des courbes indiquant une hydrolyse rapide correspondent à l'hydrolyse des parties 
amorphes des grains d'amidon, pendant que les phases à croissances lentes correspondent à l'hydrolyse des parties cristallines des grains. Ces parties qui constituent les plus dures des grains sont impénétrables aux ions $\mathrm{H}_{3} \mathrm{O}^{+}$en faible quantité dans une concentration ménagée d'acide. Il faut certainement une concentration en acide plus élevée pour augmenter la cinétique de l'hydrolyse.

\section{Digestibilité enzymatique de l'amidon natif}

Les résultats obtenus montrent que les amidons étudiés sont plus résistants au suc digestif d'escargot que celui de l'igname (N'guéssan, 2007). Cependant les résultats obtenus ici sont similaires à ceux observés sur la patate douce (N'guéssan, 2007).

\section{Teneur en amylose et en amylopectine des amidons étudiés}

Comparant les résultats obtenus à d'autres travaux antérieurs (Tetchi et al., 2007), l'amidon d'Agbablé 1 a une teneur en amylose légèrement inférieure à celle de l'igname florido $(25,3 \%)$, celle de la patate $(25,2 \%)$, Celle de l'igname kponan $(26,7 \%)$ et celle du gingembre $(26,6 \%)$. Ce même travail indique que la teneur en amylose de l'amidon de kétévie est sensiblement égale à celle de l'amidon d'une autre variété de manioc (21,6\%). Mais aussi, les amidons étudiés ici sont moins riches en amylose que celui du blé $(30,9 \%)$ (Tetchi et al., 2007). Ce qui signifie également que les amidons étudiés ici ont une utilisation dans les aliments à longue conservation plus judicieuse que celui du blé qui est un amidon de référence. Des travaux antérieurs (Whistler et BeMiller, 1997) montrent qu'il existe des amidons d'autres cultivars de manioc qui sont moins riches en amylose (17\%). D'autre part, on peut noter que l'amidon du mais est plus riche en amylose (28\%) que les amidons étudiés dans ce travail. Divers travaux ont montré diverses teneurs en amylose pour les mêmes genres de plantes (Mali et al., 2002 ; Tetchi et al., 2007). Cette observation s'expliquerait par les différences entre les conditions culturales, par les différences entre les méthodes d'extraction des lipides et par les différences entre les méthodes utilisées pour la détermination des teneurs en amylose (Mali et al., 2002).

\section{Gonflement des amidons étudiés}

Les allures observées chez les amidons de cette étude sont conforment à celle observée par Sidibé et al. (2009) chez l'amidon d'Attiéké Mossi 1. Comparativement à cette même étude menée par Sidibé et al. (2009), tous les amidons étudiés à l'exception de celui de Kétévie ont une capacité de gonflement plus élevé que celui d'Attiéké Mossi 1. Les gonflements mesurés par cette méthode directe sont largement supérieurs à ceux déterminés avec la méthode de Leach et al. (1959) qui sousestimerait cette grandeur (Sidibé et al., 2009).

\section{Viscosité des amidons étudiés}

La viscosité des amidons évolue conformément au gonflement de ceux-ci. Ce qui confirme le fait que la viscosité est la conséquence du gonflement. $\mathrm{Au}$ vu des résultats, l'amidon de TA (8) est le plus disposé à une utilisation en tant que gélifiant et épaississant dans les aliments. Car le visqueux étant un mauvais conducteur thermique, sa présence dans les aliments nécessitera une grande quantité d'énergie pour sa stérilisation. Ce qui peut conduire à la destruction de la matrice de l'aliment de base (Dillon, 1989). Aussi, la viscosité perturbe le transit digestif des aliments à cause de son opposition à l'écoulement (Bernard, 2006). Enfin, les denrées alimentaires trop visqueux entraînent une augmentation de la viscosité des contenues intestinaux, ce qui provoque des diminutions de la digestion pour les lipides et dans une moindre mesure, pour les protéines. La viscosité induit alors la 
réduction de l'absorption intestinale (Bernard, 2006). Aussi des chercheurs (Fabien et Maria, 2008) ont-ils montré que les aliments à viscosité faible génèrent plus de valeur énergétique que les aliments à viscosité élevée. C'est pourquoi la germination et la fermentation sont utilisées dans l'alimentation de nourrissons (Gopaldas et al., 1986 ; Bernard, 2006).

Dans tous les cas, pour la concentration $(4 \% \mathrm{p} / \mathrm{v})$ choisie, les viscosités des amidons étudiés ne dépassent pas celles requises pour les bouillies de sevrage (1600 cP) (Dillon, 1989). Car la viscosité de l'amidon d'Attiéké Mossi est de $317.9 \mathrm{cP}$ (cP = centiPoises).

\section{Conclusion}

Les études menées sur les amidons choisis ont permis de mettre en évidence que celui de Kétévie est le plus enclin aux agressions acide et enzymatique. Et que celui d'Attiéké Mossi 1 est le plus résistant à ces facteurs. Cette observation fait de l'amidon de Kétévie le plus intéressant en termes d'une utilisation en tant qu'excipient dans les comprimés dans l'industrie pharmaceutique. Les teneurs en amylose sont relativement équivalentes à celles observées dans d'autres amidons. L'amidon d'Agbablé 1 possède la teneur la plus élevée tandis que celui d'Attiéké Mossi 2 dispose de la plus faible teneur. Cette observation, conformément à des études préalables, fait de l'amidon d'Agbablé 1 le plus prédisposé à une forte synérèse (libération d'eau) lors d'une longue conservation, tandis que celui d'Attiéké Mossi 2 est le moins prédisposé à la synérèse. La détermination du gonflement selon la méthode directe démontre que l'amidon d'Agbablé 1 présente la plus grande capacité d'absorption d'eau tandis que celui de Kétévie gonfle le moins. Au niveau de la viscosité, la plus élevée est observée chez l'amidon d'Attiéké Mossi 2 et la moins élevée, chez celui de TA (8).
Avec les résultats obtenus dans la présente étude l'étape à venir est celle de l'implication réelle de ces amidons dans les différentes voies d'utilisation prédites par les investigations physicochimiques réalisées. Il serait en effet important dans les travaux futurs d'impliquer les amidons de ces 4 variétés de manioc dans la fabrication de bioplastiques alimentaires, dans la fabrication de gelées et sauces afin de confirmer ou infirmer les prédictions faites ici.

\section{REMERCIEMENTS}

Docteur FOFANA Alhassan, pour son aide lors des manipulations.

Docteurs GOLI Zohouri Pierre, NZUE Boni et Mme DJEDJI Cathérine Ebah, tous chercheurs au CNRA, pour nous avoir octroyé le matériel biologique.

\section{REFERENCES BIBLIOGRAPHIQUES}

Adrian J, Régine F. 1996. Nature et Propriétés de l'Amidon. Partie Technique. INIST CNRS; 17-21.

Amani NG. 1993. Contribution à l'étude des tubercules de taro (Xanthosoma sagittifolium L. Schott): évolution des propriétés physicochimiques de l'amidon au cours destraitements technologiques. Doctorat, Université Nationale de Côte d'Ivoire, pp. 62-103.

Amani NG, Tetchi FA, Coulibaly A. 2004. Propriétés physicochimiques de l'amidon de gingembre (Zingiber officinale roscoe) de Côte d'Ivoire. Tropicultura, 22(2): 7783.

AOAC. 1975. Officials methods of analysis association of agricultural chemistry: Washington D.C.

Bernard C. 2006. Les désordres digestifs générés par les blés chez le poulet en croissance peuvent être résolus par les sélections génétiques végétale et animale. Département INRA «PHASE» et «génétique animale». CIRAD de Montpellier. 
Chrastil J. 1987. Improved colorimetric determination of amylose in starches or flours. Carbo. Research, 159: 154-158.

Davies EM, Maryke TL, Elizma K, Ibrahim RMB, John DKS. 2008. Some properties of starches from cocoyam (Colocasia esculenta) and cassava (Manihot esculenta Crantz.) grown in Malawi. Afr. J. Food Sci., 2: 102-111.

Dillon JC. 1989. Les Produits Céréaliers dans L'alimentation de Sevrage du Jeune Enfant en Afrique. Céréales en Régions Chaudes. AUPELF-UREF, Eds John Libbey Eurotext : Paris ; 299-307.

Dreher ML, Dreher CJ, Berry JW. 1984. Starch digestibility of foods: a nutritional perspective. C.R.C. Critical Rev. Food Sci. Nutri., 20: 47-71.

Dubois M, Gilles KA, Hamilton JK, Rebers PA, Smith F. 1965. Colorimetric method for determination of sugars and related substances. Analytical Chem., 28: 350356.

Fabien S, Maria V. 2008. Effet d'une xylanase sur la valeur alimentaire du blé et de ses coproduits, drêches et son, chez le porc charcutier. Arvalis (institut du végétal). newsl@lim. $\mathrm{N}^{\circ} 18$, p. 1 et 2.

FAO. 1998. Archives (amidon).

Freitas RA, Paula RC, Feitosa JPA, Rocha S, Sierakouski M-R. 2004. Amylose contents, rheological and gelatinization kinetics of yam (Dioscorea alata) and cassava (Manihot utilissima) starches, Carbo. Polym., 55: 3-8.

Gallant DJ, Bewa H, Buy QH, Bouchet B, Szylit O, Sealy L. 1982. An ultrastructural and nutritional aspects of some tropical tuber starches. StarchStärke, 34: 255-262.

Gopaldas T, Mehta PA, Gandhi H. 1986. Studies on the reduction in viscosity of thick rice gruel with small quantities of an amylase-rich ceral malt. Food Nutri. Bull., 8: 42-47.
Hoover R, Vasanthan T. 2004. Effect of heatmoisture on the structure and physicochemical of cereal, legume and tuber starches, Carbohydrate Research, 252: 22-53.

Karim AA, Toom LC, Lee VP, Ong WY, Fazilah A, Noda T. 2007. Effects of phosphorus contents on the gelatinization and retrogradation of potato starch. $J$. Food Sci., 72(2): 132-138.

Kouakou KM. 2004. Etude des propriétés physico-chimiques des amidons de Bonoua 2, Bonoua akpessé et du 524. DEA. UAA. Abidjan. Côte d'Ivoire, p. 33-35.

Lagarrigue S, Alvarez G, Cuvelier G, Flick D. 2008. Swelling kinetics of waxy maize and maize starches at high temperatures and heating rates. Carbo. Polym., 73: 148-155.

Leach HW, MCcowen LD, Schoch JJ. 1959. Structure of the starch granule swelling and solubility patterns of various starches. Cereal Chem., 36: 534-544.

Mali S, Grossmann VE, Garcia MA, Martino MN，Zaritzky NE. 2002. Microstructural characterization of yam starch films. Carbo. Polym., 50: 379-386.

Massaux C, Bodson B, Lenartz J, Sindic M, Sinnaeve G, Dardenne P, Falisse A, Deroanne C. 2006. L'amidon natif du grain de blé : un composé naturel à valoriser par la connaissance de ses propriétés techno-fonctionnelles, Livre blanc «céréales » F.U.S.A. et CRA-W Gembloux, pp. 3 et 5 .

N'guessan G. 2007. Etude des propriétés physiques et fonctionnelles des amidons d'Ipomea batatas et de Dioscorea cayenensis rotundata. Thèse d'Etat en pharmacie. Université de Cocody. Abidjan. Côte d'Ivoire, pp. 45- 79.

Schmitz CS, De Simas KN, Jao JJ, De Mello CARD, Amante ER. 2006. Cassava starch functional properties byetherification- 
hydroxypropylation. Int. J. Food Sci. Tech., 41: 681-687.

Sea BT, Okou WO, Saki JS, Sako A, Sidibé D, Golly J, Otchoumou A. 2008. Hydrolyse enzymatique des épluchures de manioc (Manihot esculenta Crantz) par le suc digestif de l'escargot limicolaria flammea. Rev. CAMES, série A(07): 7-9.

Sidibé D, Sako A, Agbo NG. 2005. Etude de quelques propriétés physico chimiques des amidons de cinq (5) variétés de manioc (Attiéké Mossi 1, Attiéké Mossi 2, Agbablé1, Kétévie et TA (8) cultivées en Cote d'ivoire. Rév. CAMES, série A(05): 92-98.

Sidibé D, Sako A, Dally LI, Ezoua P, Chatigre K. 2009. Thermal effects on granules and direct determination of swelling capacity of starch from a cassava cultivar (Attiéké
Mossi 1) cultivated in Côte D'Ivoire. Afr. J. Biotech., 8(15): 3615-3622.

Tang H, Mitsunaga T, Kawamura Y. 2005. Functionality of starch granules in milling fractions of normal wheat grain. Carbo. Polym., 59: 11-17.

Tetchi FA, Sabatié AR, Amani NG, Colonna P. 2007. Molecular and physicochemical characterization of starches from yam, cocoyam, cassava, sweet potato and ginger produced in the Ivory Coast, J. Sci. Food Agric., 87: 1906-1916.

Thire RMSM, Simao RA, Andrade CT. 2003. High resolution imaging of the microstructure of maize starch films. Carbo. Polym., 54(2): 149-158.

Whistler RL, BeMiller JN. 1997. Carbohydrate Chemistry for Food Scientists. St. Paul Eagan Press; 117-151. 